\title{
The effective diagnostic process may shorten TISD and improve overall survival of young patients with colorectal cancer
}

\author{
Mo-Jin Wang • Zi-Qiang Wang • Rui Wang • \\ Zong-Guang Zhou
}

Accepted: 27 April 2014 / Published online: 6 May 2014

(C) Springer-Verlag Berlin Heidelberg 2014

Dear Editor:

We have read the recent publication "Do young patients have different clinical presentation of colorectal cancer causing delay in diagnosis?" by Sousa et al. (Int $J$ Colorectal Dis. 2014; 29:519-527) with great interest. Sousa et al. reported that the time interval between onset of symptoms and diagnosis (TISD) was shorter in the young than older colorectal cancer patients. We think the following reasons might explain these results contrast with their initial hypothesis. In this research, compared with actual situation in the population, the young patients who go to their reference university hospital may have stronger self-health care consciousness, more complete medical insurance, and more convenient way to see doctors. When having clinical symptoms, they are more proactive in seeking medical care and avoid delayed diagnosis. On the other hand, a part of the older patients included this research, because of the mobility problems and so on, does not take routine surveillance or screening, leading to the delayed diagnosis frequently at the later stages of disease. Therefore, the conclusion that "young patients with colorectal cancer had clinical and pathological presentation similar to that of older patients" should be cautiously regarded. Just as Connell et al. (Am Surg. 2003; 69:866-872.) and Ahnen et al. (Mayo Clin Proc. 2014; 89:216-224) described, the effective diagnostic process of symptomatic young patients with colorectal cancer may shorten TISD and improve overall survival.

Sincerely,

Mo-Jin Wang, Zi-Qiang Wang, Rui Wang, and ZongGuang Zhou
M.-J. Wang $(\bowtie) \cdot$ Z.-Q. Wang $\cdot$ Z.-G. Zhou

Department of Gastrointestinal Surgery, West China Hospital,

Sichuan University, 37 Guo Xue Xiang, 610041 Chengdu, China

e-mail: wangmojin2001@163.com

R. Wang

Department of Gastroenterology, West China Hospital, Sichuan

University, 37 Guo Xue Xiang, 610041 Chengdu, China 